\title{
Visible Light Source Temperature Estimation Using Digital Camera Photography
}

\author{
*ANAGHA M. PANDITRAO, ** PRITI P. REGE \\ *Cummins College of Engineering for Women, ** College of Engineering, Pune \\ INDIA
}

\begin{abstract}
Light source temperature measurement has been an important aspect in many industrial applications. The aim of this study is to determine the temperature of different sources like flames, incandescent lamps. The sensor installation is difficult in these sources. Imaging these sources to correlate with the temperature is attempted. A digital still camera is used to capture the source images. The optical characteristics of CCD sensor are not mentioned in the camera specifications. It is desired that the device bandwidth should be greater than the source bandwidth. Hence, the optical characteristics of the camera are also obtained. As Incandescent lamp is the most familiar light source its photographs are taken. The images are taken at known excitation voltages in a dark room with camera settings unaltered. Filament temperature is a function of intensity. Intensity difference of two consecutive images is found out by image subtraction. Unwanted part of image, like reflection from the shell is removed by thresholding and segmentation. The colour temperature of the filament can be found out with reference to black body radiations. If these values are compared with standard temperatures, source temperature values can be predicted.
\end{abstract}

Keywords: Light Source Imaging, Non-contact Temperature Measurement, Digital Still Camera

\section{Introduction}

This study deals with the determination of temperature in sources like furnace, incandescent lamp. The installation of sensor in above-mentioned sources is difficult. If the image of these sources is acquired by some means and post processing is carried out it is possible to predict the temperature. Most of the light sources emit light as a function of temperature. The light emitted by an incandescent source is a mixture of light with different wavelengths. The light from a black body is a mixture of light with a continuous range of wavelengths. An incandescent lamp is very nearly a blackbody radiator. This is the most commonly used light source. The distribution of power in the wavelengths it produces can be described by the temperature of a black body radiator whose light would appear to the human eye to be of the same colour [1]. The development of digital cameras has opened up possibilities for powerful new diagnostic techniques employing two-dimensional imaging. Digital still cameras (DSCs) have gained significant popularity in recent years [2]. Digital photography is the easily available fast technique and requires no other sensor. It is also possible to acquire the image to get total temperature distribution. Qualitative visualization for the temperature measurement is done using digital camera [3]. A SONY make Digital Still Camera DSC-S60 is used to acquire images. The optical characteristics of CCD sensor are not mentioned in the camera specifications.

The proposed work is to obtain the characteristics of the digital camera and to obtain filament temperature of incandescent lamp by image processing.

\section{Camera Characteristics}

In the proposed work a SONY make Still Digital Camera DSC-S60 is used to capture the images of various sources. As the optical characteristics are not mentioned in the specifications, different sources are used to cover the wide bandwidth ranging from IR to UV. It is desired that the device bandwidth should be greater than the source bandwidth. To verify if the camera is having adequate bandwidth, it is necessary to plot the camera characteristics. 


\subsection{Experimental setup:}

While designing the setup it is ensured that the effect of stray light is minimized and reflection of light is avoided. We have used following sources for our experimentation :

- $\quad 5 \mathrm{~W}, 12 \mathrm{~V}$ Lumina make tungsten filament lamp

- LED: Colour (R, G, B), UV and IR

Enclosure length is adjusted so as to match camera minimum distance specifications. The setup photographs are shown in Figure1
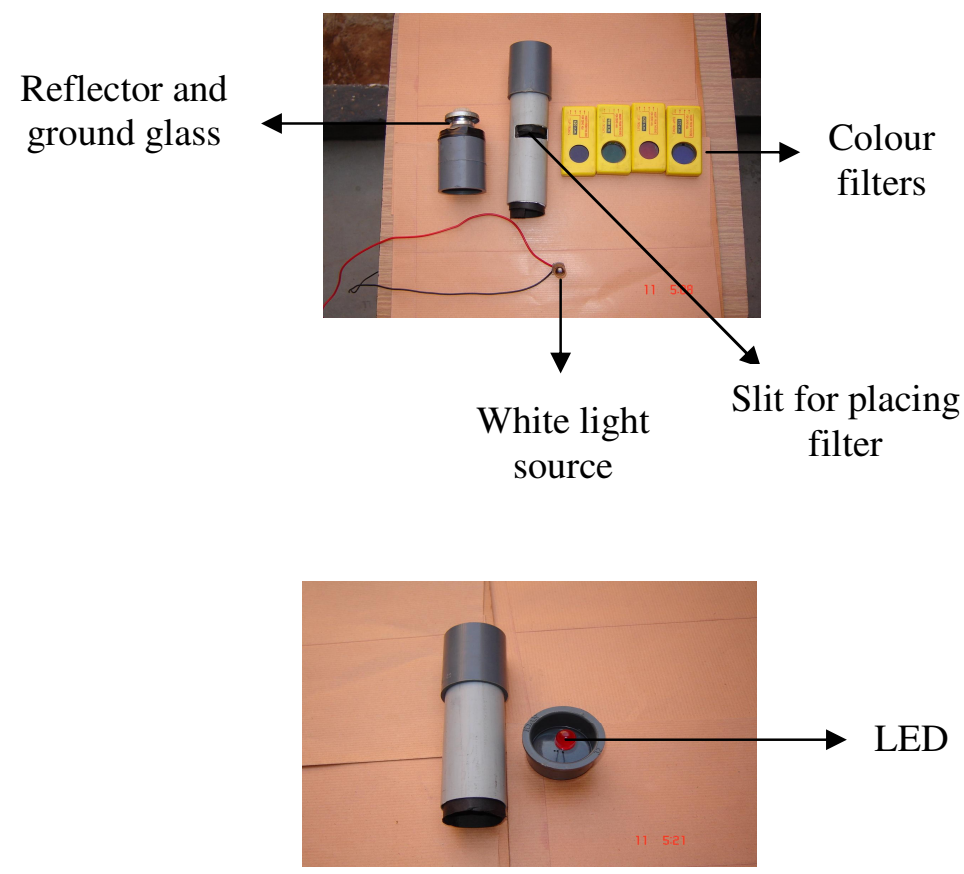

Figure1: Setup Photographs

Photographs of colour filters (Red, Green, and Blue) are taken where the source intensity is changed without affecting its white light nature. Similar technique is applied for LED array (Red, Green, Blue).

The images obtained are shown in Figure 2

\subsection{Images obtained:}

Source: Filter

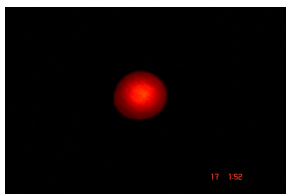

Red

Source: LED

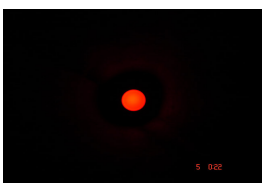

Red

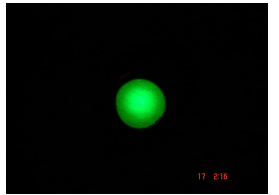

Green

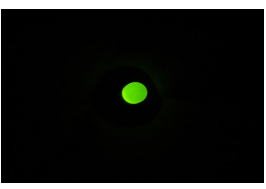

Green

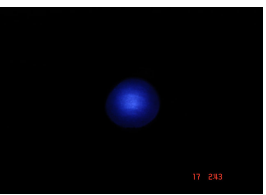

Blue

\section{Figure 2: Filter and LED Images}

\subsection{Processing}

The colour values (Red, Green, and Blue) of the images acquired are separated as shown in Figure 2. The values are compared with standard digital values. Red coloured images are compared with $(255,0,0)$, green and blue are compared with $(0,255,0)$ and $(0,0,255)$ respectively. The processing is carried out using MATLAB software.

After processing the images and comparing the colour values with the standard values, it is observed that the obtained values are fairly matching with the standard values for $\mathrm{R}, \mathrm{G}$, and $\mathrm{B}$.

\section{Source Image Acquisition}

After obtaining the characteristics of camera, images of lamp filament are taken in a dark room with known excitation voltages. Figure 3 shows a filament image at a typical excitation voltage. In the photograph, section (a) is the filament image and section (b) is filament reflection from shell. 


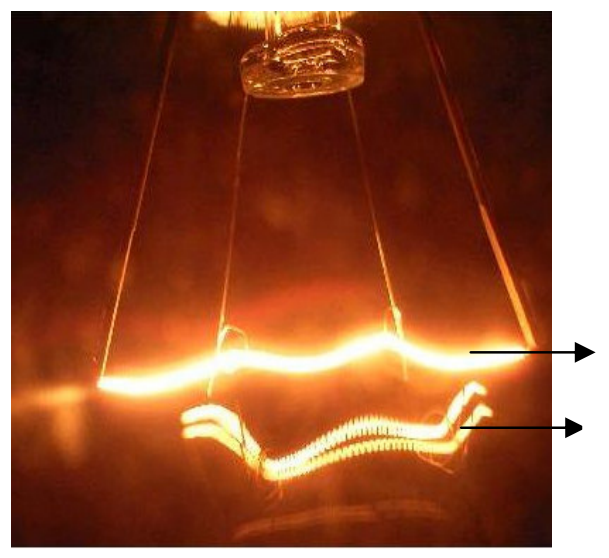

Figure 3: Incandescent Lamp Filament Image

The block schematic of the proposed system is given in Figure 4

Light Source

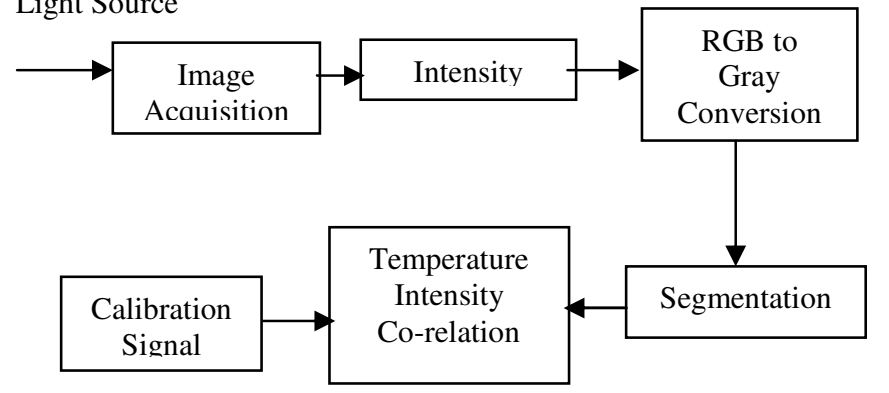

Figure 4: Block Schematic of the Proposed Noncontact Source Temperature Measurement System

Incandescent and fluorescent lights are two most common sources of artificial light. Incandescent light is created by passing an electric current through a resistance tungsten filament [3]. Characteristics of light can be described by the temperature. An incandescent lamp is connected to a dimmer. As the dimmer is turned up, the voltage increases and the lamp's filament becomes warmer and warmer until it begins to glow cherry red. As the voltage continuous to increase, the filament gets hotter and hotter, glows more brightly and less and less red [1]. The filament images of a $40 \mathrm{~W}$ Philips make lamp are captured on digital camera. Incandescent lamps emit light solely because of their temperature. Tungsten wire temperature is function of flow of electric current [4]. To avoid ambient light effect, the images are taken in a dark room. Images acquired using digital camera are used in further processing. Camera settings are not changed till all images are captured.

The graph of RMS value of intensity Vs the excitation voltage is shown in Figure 5. The intensity varies linearly with excitation voltage. The images obtained are converted to gray for further processing. Unwanted parts are removed using segmentation algorithm. The change in colour or intensity can be corelated with the filament temperature [5]. The temperature values can be compared with standard temperatures.

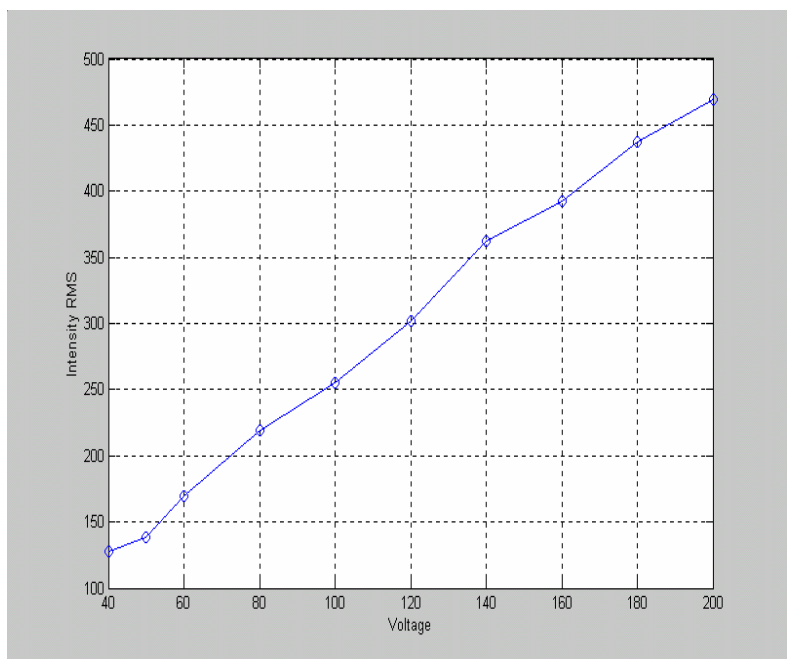

\section{Figure 5: Graph of Intensity RMS Value Vs Excitation Voltage}

\section{Image Processing}

The images acquired using a digital camera of known resolution, are having natural colour components Red, Green and Blue [6]. The intensities of two consecutive images are compared to view the change with respect to excitation voltage. Figure 6 shows this intensity comparison of images at $100 \mathrm{~V}$ and $120 \mathrm{~V}$.

These RGB components are converted to gray scale for further processing. To find out the intensity difference, images are converted to matrix form. The intensity component of every image is separated [6]. By subtracting the intensity matrices of two consecutive gray images intensity difference is obtained. 

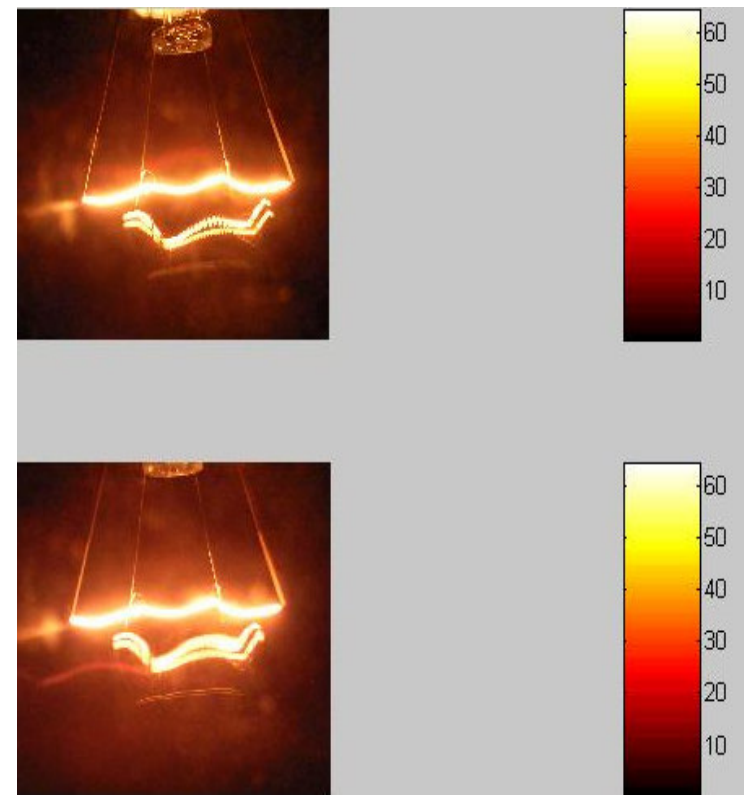

\section{Figure 6: Intensity Comparison of Two Consecutive Images}

Figure 7 shows the difference between two images. Images (a) and (b) are the images at excitation voltage $200 \mathrm{~V}$ and $230 \mathrm{~V}$ respectively. Image (c) is the intensity difference between above-mentioned images.

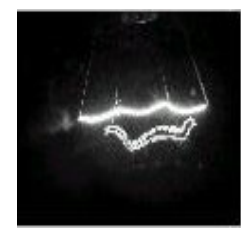

(a)

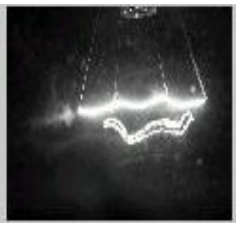

(b)

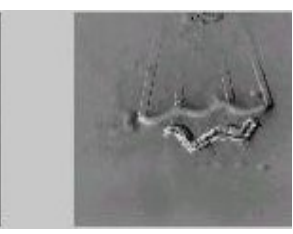

(c)

\section{Figure 7: Intensity Difference Between Two Images}

In the images under study, along with the filament image, shell reflection is also seen. Hence, preprocessing of the images is required. In the analysis of area of interest in image, it is essential to distinguish between the object of interest i.e. filament from the rest. Most commonly used techniques for segmenting foreground from the background are thresholding and edge detection. No segmentation technique is perfect and there is no universally applicable technique that works for all images [8]. Because of its intuitive properties and simplicity of implementation, image thresholding enjoys a central role in this application.

The proposed work is based on histogram-based thresholding. The brightness histogram of an image at typical excitation is shown in Figure 8. In this histogram $\mathrm{X}$-axis represents the intensity of the pixels whereas $\mathrm{Y}$ axis represents number of pixels having same intensity values.

The histogram of the image is examined for locating peaks and valleys. A parameter ' $\theta$ ' called threshold is chosen and applied to the image a [m, n] as follows:

$$
\begin{array}{ll}
\text { If } \quad \mathrm{a}[\mathrm{m}, \mathrm{n}] \geq \theta & \mathrm{a}[\mathrm{m}, \mathrm{n}]=\text { Lamp Filament }=1 \\
\text { Else } & \mathrm{a}[\mathrm{m}, \mathrm{n}]=\text { Background }=0
\end{array}
$$

The threshold is chosen from the brightness histogram of the region that is expected to segment [9].

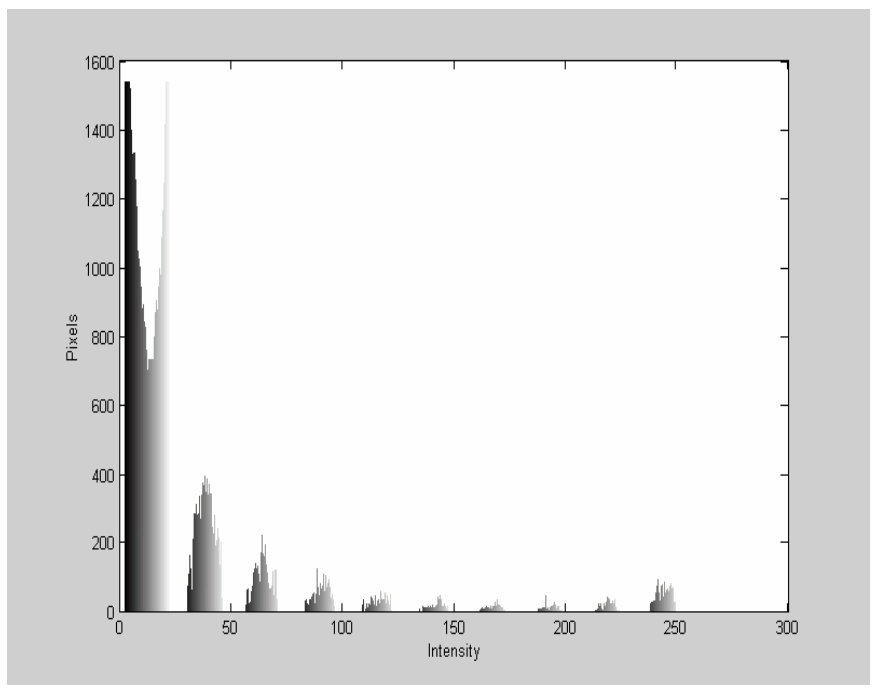

\section{Figure 8: Intensity Histogram of Image At Typical Excitation}

Following observations are made after studying the brightness histogram:

The histogram is multimodal. The largest peak in the histogram depicts the background and smaller ones represent filament and reflection from shell. The peaks at higher brightness representing the filament are the objects of interest. 
A threshold is selected iteratively by examining local neighborhood histogram. This threshold value is used to filter out unwanted data. The filament images obtained after thresholding are shown in Figure 9. Image (a) and (b) are the filament images at $100 \mathrm{~V}$ and $120 \mathrm{~V}$ respectively, obtained after thresholding.

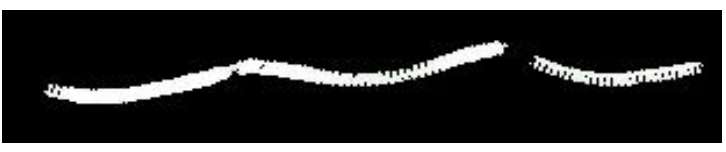

(a)

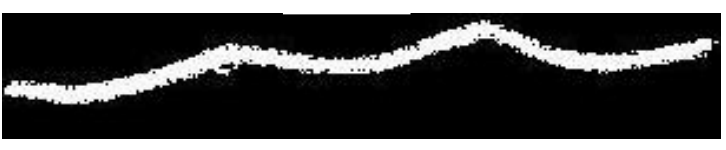

(b)

Figure 9:Filament Images After Thresholding

Intensity difference is observed in the above filament images. Filament temperature changes with change in intensity. The colour temperature of the filament can be found out with reference to black body radiations [9]. According to Stefan-Boltzmann's law, the energy emitted by a black body per unit area and unit time is proportional to the power "four" to the absolute temperature of the body. The "grey" body is represented by the filament of an incandescent lamp whose energy emission is investigated as a function of a temperature. The energy emitted per unit area and unit time at temperature $\mathrm{T}$ and wavelength $\lambda$ within the interval $\mathrm{d} \lambda$, is designated by $\mathrm{dL}(\mathrm{T}, \lambda) / \mathrm{d} \lambda$, Plank's formula states:

$$
\frac{\mathrm{dL}(\mathrm{T}, \lambda)}{\mathrm{d} \lambda}=\frac{2 \mathrm{c}^{2} \mathrm{~h} \lambda^{-5}}{\exp (\mathrm{hc} / \lambda \mathrm{kT})-1}
$$

where, $\mathrm{c}=$ velocity of light $\left(3 \times 10^{8} \mathrm{~m} / \mathrm{s}\right)$,

$\mathrm{h}=$ Plank's constant $\left(6.62 \times 10^{-34}\right)$, $\mathrm{k}=$ Boltzmann's constant $\left(1.381 \times 10^{-23}\right)$.

Integration of this gives the relation of intensity and temperature i.e. Stefan-Boltzmann's law.

$$
\begin{aligned}
\mathrm{L}(\mathrm{T}) & =\frac{2 \pi 5 \mathrm{k}^{4} \mathrm{~T}^{4}}{15 \mathrm{c}^{2} \mathrm{~h}^{3}} \\
& =\sigma \mathrm{T}^{4}
\end{aligned}
$$

where, $\sigma=5.67 \times 10^{-8}$

On the basis of these laws temperature can be related with the intensity of the source. Temperature-intensity co-relation is shown in Figure 10. If these temperature values are calibrated using standard, filament temperature can be predicted [9].

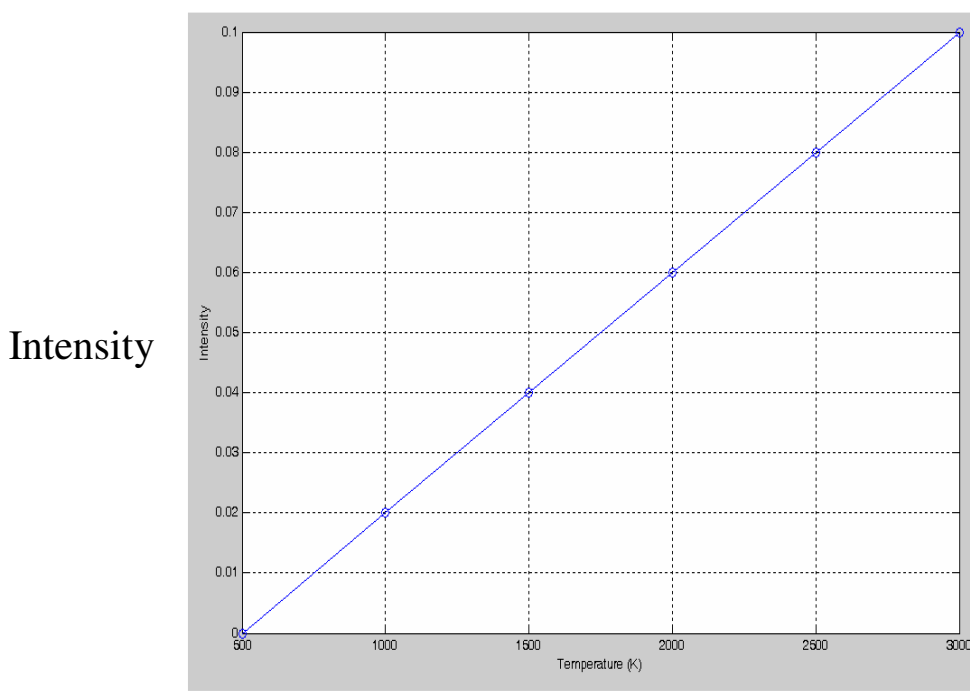

Temperature (K)

Figure 10: Intensity Temperature Co-relation

\section{Results And Conclusion}

The optical characteristics of the camera used for image capturing are obtained. From these results it is observed that the digital camera used to capture source images is having enough bandwidth to cover visible range (400$700 \mathrm{~nm}$ )

Filament images of a typical $40 \mathrm{~W}$ Incandescent Lamp are captured using digital camera of known resolution. Desired image segment is selected for processing using thresholding. Intensity change can be correlated with temperature. Using standard, temperature intensity relation is established. Using this relationship for a known intensity value filament temperature can be predicted.

\section{References}

[1] Mike Wood, Color temperature of metal halide sources, Aug. 2001 
[2] Rajeev Rananath, Wesley Snyder, Youngjun Yoo, Marks Drew, "Color Image Processing”, IEEE Signal Magazine, pp.16-20, Jan 2005

[3] Moritz Stoerring, Light spectra and colour temperature, July 99

[4] http://www.wartsila-nsd.com

[5] www. Color phenomena.com

[6] Gonzalez, Woods, Digital Image Processing, Pearson Educatio, Singapore, 2003.

[7] Anil K. Jain, Fundamentals of Digital Image Processing, Prentice Hall Private Limited, New Delhi, 2001.

[8] Dr. Horace, Dr. Diggang Shen, An active surface paradigm for adaptive image thresholding, 2002

[9] Dr. James C. Tilton, "Hierarchical Image Segmentation", NASA's Goddard space flight center

[10] J. W. Dooley, "Black Body Radiation and Characteristics of Incandescent Lamp”, 2002 